Published online: 28 August 2020

https://doi.org/10.1038/s41591-020-1062-6

References

1. Equality and Human Rights Commission. Tackling racial harassment: universities challenged. https://www. equalityhumanrights.com/en/publication-download/ tackling-racial-harassment-universities-challenged (2020).

2. Eichbaum, Q. G. et al. Acad. Med. https://doi.org/10.1097/ ACM.0000000000003473 (2020).
3. Khan, M. S. et al. Lancet 393, 594-600 (2019).

4. Boum Ii, Y. et al. BMJ Glob. Health 3, e000868 (2018).

5. Abimbola, S. BMJ Glob. Health 4, e002068 (2019).

Acknowledgements

The authors acknowledge LSHTM's DGH group and the BLM network of current and former staff and students. The authors also acknowledge and thank L. Hirsch for her research on colonial history of LSHTM, which inspired this Correspondence.
Author contributions

N.A.E., D.P. and M.S.K. conceived the idea together. N.A.E developed the first draft, M.S.K. and D.P. made significant revisions to the draft, and all authors approved the final version for publication.

Competing interests

The authors declare no competing interests.

\title{
Controlling the COVID-19 pandemic in Brazil: a challenge of continental proportions
}

To the Editor - Five months into the COVID-19 epidemic, as of 19 August 2020, Brazil reported a total of $3,407,354$ PCR-confirmed cases and 109,888 deaths due to COVID-19, a case fatality rate (CFR) of $3.2 \%{ }^{1}$. These numbers likely reflect a massive underestimate as individuals who are asymptomatic, pauci-symptomatic or pre-symptomatic are often not tested, while contact tracing remains limited ${ }^{2}$. The rapid spread of COVID-19 in Brazil is attributable to many factors, including urban density, timing of the implementation and maintenance of social distancing policies, and limited testing capacity. Furthermore, as of late June 2020, as a result of economic and political pressures, many Brazilian states have lifted restrictions on businesses and shared spaces despite increasing numbers of cases.

Fueling the Brazilian pandemic is the fact that a large proportion of the population live in medically underserved and dense communities, including urban slums characterized by limited health care access and poor sanitation. Discontinuation of social distancing measures disproportionately affects vulnerable communities, where transmission of COVID-19 is already considerable, as many residents are unable to self-isolate because they must work. As past modeling studies of influenza epidemics demonstrate, slum areas sustain higher transmission rates than non-slum areas under all intervention scenarios because of crowding and higher household sizes ${ }^{3}$. In addition, oversaturated healthcare systems often turn patients away, increasing the number of preventable deaths, particularly in vulnerable communities.

We implemented three susceptibleinfected-exposed-recovered (SEIR) compartmental epidemiological models (Delphi, Squire and YYG) for which source code is publicly available to forecast the future trajectory of the epidemic in Brazil ${ }^{4-6}$. The models were parameterized with COVID19 cases and mortality from 26 February to 11 August 2020 according to data from the Brazilian Ministry of Health. The models constructed with these data were projected forward to 1 January 2021. Each of the three models produced predictions of the number of cases and deaths per day. The mortality and incidence predictions varied somewhat among models. To produce an elementary summary of these outputs, we calculated the mean and range of the three predictions.

The mean prediction of the models is that by January 2021, with social distancing measures in place, the number of confirmed COVID-19 cumulative cases in Brazil will be 5 million with 161,212 deaths (which constitutes a predicted CFR of 3.2\%). Delphi predicted 117, 377 deaths and 3.7 million cases, YYG 182,147 deaths and 4.7 million cases, and Squire 184,113 deaths and 6.6 million cases. While valuable, the aim of the models reported here is to predict the first wave of the epidemic. As such, they do not incorporate a number of other epidemiological processes that could potentially have a considerable effect on the number of cases, such as waning immunity, reinfection, recruitment of new susceptible individuals, or vaccination, though more complex compartmental models include such processes ${ }^{7,8}$. The models also do not take into account population behaviors triggered by political mistrust that can often fuel the COVID-19 pandemic, a phenomenon termed the Cummings effect in the United Kingdom?.

The aforementioned models represent one of a number of predictive approaches that can be marshaled to estimate the size of the epidemic in Brazil. Another approach is to assess how the rollback of social distancing could influence the size. For instance, a lack of physical distancing can lead to a fivefold increase in acquisition risk ${ }^{10}$. If precautionary measures such as social distancing were suspended, a potentially catastrophic fivefold escalation in the number of cases could occur, with a projection of up to 28 million cases and 902,787 deaths (3.2\% CFR) by January 2021 in Brazil if no other mitigation measures were put in place or if vaccine efforts were not implemented on a nationwide scale. In this worst-case scenario, approximately $13.2 \%$ of the Brazilian population of 212 million could become infected with SARS CoV-2 while traditional models predict approximately $2.4 \%$ could become infected.

Regardless of the models used to predict cases and mortality, the pandemic has unquestionably taken an unprecedented economic toll on Brazil, in addition to the overwhelming disease burden. It is crucial, however, to carefully plan easing of social distancing measures in a country of continental proportions to minimize as much as possible the unfathomable cost in human lives.

\section{Data availability}

Data used for this analysis are publicly available from the Brazilian Ministry of Health Coronavirus Panel at http://covid. saude.gov.br.

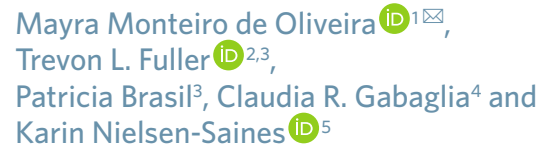


${ }^{1}$ Faculdade de Odontologia da Universidade de Sāo Paulo (USP), Sāo Paulo, Brazil. ${ }^{2}$ Institute of the Environment \& Sustainability, University of California Los Angeles, Los Angeles, CA, USA. ${ }^{3}$ Laboratorio de Doenças Febris Agudas, Instituto Nacional de Infectologia, Fundação Oswaldo Cruz, Rio de Janeiro, Brazil. ${ }^{4}$ Biomedical Research Institute of Southern California, Oceanside, CA, USA. ${ }^{5}$ Division of Pediatric Infectious Diseases, David Geffen School of Medicine at UCLA, Los Angeles, CA, USA.

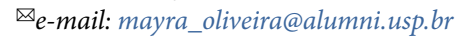

Published online: 4 September 2020 https://doi.org/10.1038/s41591-020-1071-5

\section{References}

1. Center for Systems Science and Engineering (CSSE) at Johns Hopkins University. COVID-19 dashboard. https:// www.arcgis.com/apps/opsdashboard/index.html\#/ bda7594740fd40299423467b48e9ecf6 (2020).

2. Lau, H. et al. Pulmonology https://doi.org/10.1016/j. pulmoe.2020.05.015 (2020).

3. Adiga, A. et al. BMJ Open 8, e017353 (2018).

4. Li, M. L. et al. medRxiv https://doi. org $/ 10.1101 / 2020.06 .23 .20138693(2020)$

5. Watson, O.J., Winskill, P., Walker, P.G. \& Whittaker, C. Squire. SEIR transmission model of COVID-19. https://mrc-ide.github. io/squire/ (2020).

6. Youyang, G. COVID-19 projections using machine learning. https://github.com/youyanggu/covid19_projections (2020).

7. Kissler, S. M., Tedijanto, C., Goldstein, E., Grad, Y. H. \& Lipsitch, M. Science 368, 860-868 (2020).
8. Jansson, J. COVID-19 modelling is wrong. Medium https://medium.com/@jamesjansson/covid-19-modell ing-is-wrong-f7246e3dc396 (2020)

9. Fancourt, D., Steptoe, A. \& Wright, L. Lancet 396, 464-465 (2020) 10. Chu, D. K. et al. Lancet 395, 1973-1987 (2020).

Author contributions

M.M., T.F., C.R.G, P.B. and K.N.S performed the literature review. T.F. performed the dataset analysis. M.M., T.F. and K.N.S. interpreted the data. M.M., T.F. and K.N.S wrote the first draft of the manuscript. All authors participated in critical review of the manuscript and contributed to the final manuscript product.

Competing interests

The authors declare no competing interests. 